ZOOLOGIA 27 (3): 408-412, June, 2010

doi: $10.1590 /$ S1984-46702010000300013

\title{
Two new species and new records of Syntrechalea (Araneae: Lycosoidea: Trechaleidae) from Brazil
}

\author{
Estevam L. C. da Silva \& Arno A. Lise \\ Laboratório de Aracnologia, Museu de Ciências e Tecnologia, Pontifícia Universidade Católica do Rio Grande do Sul. Avenida \\ Ipiranga 6681, Prédio 40, Sala 125, $90619-900$ Porto Alegre, Rio Grande do Sul, Brazil. E-mail: estevamsilva@gmail.com
}

\begin{abstract}
Currently, nine species of Syntrechalea F.O. Pickard-Cambridge, 1902 are known, six of them occurring in Brazil. In this study, two new species of of this genus are described and illustrated from the states of Amazonas and Tocantins, northern Brazil. Syntrechalea neblina sp.nov. can be distinguished from all known species by the bifurcated guide of the median apophysis. Syntrechalea robusta sp.nov. can be distinguished from all known species by the wider and concave ectal division of the retrolateral tibial apophysis. Additionally, new records of S. adis Carico, 2008, S. brasilia Carico, 2008, S. caporiacco Carico, 2008, S. napoensis Carico, 2008, S. syntrechaloides (Mello-Leitão, 1941) and S. tenuis F.O. Pickard-Cambridge, 1902 are provided.
\end{abstract}

KEY WORDS. Distribution; Neotropical region; spider; taxonomy.

The spider genus Syntrechalea F.O. Pickard-Cambridge, 1897 was recently revised by CARICO (2008) and five new species from South America were described and illustrated. SILVA \& Lise (2008) described and illustrated a new species from Colombia, S. colombiana (known only by the male). Silva \& LISE (2009) also described the male of Syntrechalea reimoseri (Caporiacco, 1947) from Cusco, Peru. Currently, nine species of Syntrechalea are known and most of its representatives are apparently restricted to South America, except by $S$. tenuis F.O. Pickard-Cambridge, 1902 (type-species), which occurs from Mexico to Colombia (Platnick 2009).

The representatives of this genus have an unusual habitat preference. Different from other trechaleid, that usually occur in rocky margins of streams (CARICO 1993), Syntrechalea spp. occur mainly in tree trunks near the water and have adaptations to the arboreal foraging habitat: the body is flattened, cephalic area elevated, legs are long and slender, with both tarsi and metatarsi flexible, and a with large number of macrosetae pairs on the tibia of legs I and II (CARICO 2008).

This work describes and illustrates two new species of Syntrechalea from northern Brazil. Additionally, new records of Syntrechalea adis Carico, 2008, S. brasilia Carico, 2008, S. caporiacco Carico, 2008, S. napoensis Carico, 2008, S. syntrechaloides (Mello-Leitão, 1941), and S. tenuis are provided.

\section{MATERIAL AND METHODS}

The material examined is deposited in Instituto Butantan, São Paulo, Brazil (IBSP, A.D. Brescovit), Intituto Nacional de Pesquisas da Amazônia, Manaus, Brazil (INPA, C. Magalhães), Museu de Ciências e Tecnologia da Pontifícia Universidade
Católica do Rio Grande do Sul, Porto Alegre, Brazil (MCTP, A.A. Lise), and Museu Paraense Emílio Goeldi, Belém, Pará, Brazil (MPEG, A.B. Bonaldo). The nomenclature of the male palpus and female epigynum structures follows CARICO (1993, 2008) and SILVA \& LISE $(2008,2009)$. To study the excised epigyna, the soft tissue was removed by a combination of dissection with a small surgical blade and immersion in the enzyme tripsine for 48 hours at $25^{\circ} \mathrm{C}$ to remove the soft tissue. The scanning electron micrographs (SEM) were made using a Philips XL 30 of the Centro de Microscopia e Microanálises (CEMM) of the Pontifícia Universidade Católica do Rio Grande do Sul (PUCRS). All the measurements are in millimeters. The abbreviations related to eye measurements, including diameter, interdistances, and median ocular quadrangle, are those routinely used in spider descriptions.

\section{TAXONOMY}

\section{Syntrechalea neblina sp. nov.}

Figs 1-5

Types. Male holotype from Pico da Neblina, Cachoeira do Tucano, São Gabriel da Cachoeira, state of Amazonas, Brazil $\left(00^{\circ} 07^{\prime} \mathrm{N}, 67^{\circ} 05^{\prime} \mathrm{W}\right), 22 . \mathrm{IX} .2007$, A. Nogueira leg, deposited in INPA. Paratype: one female, same locality as holotype, 23.IX.2007, N.L.M. Hung leg. (INPA).

Diagnosis. The male of $S$. neblina sp. nov. is similar to the one of $S$. tenuis (CARICo 2008: figs 8-9) by the shape of the retrolateral tibial apophysis but can be distinguished by the bifurcated guide of the median apophysis (Figs 1 and 2). The female is similar to those of $S$. tenuis (CARICo 2008: 123, figs 1011 ) by the general shape of the middle field and anterior por- 

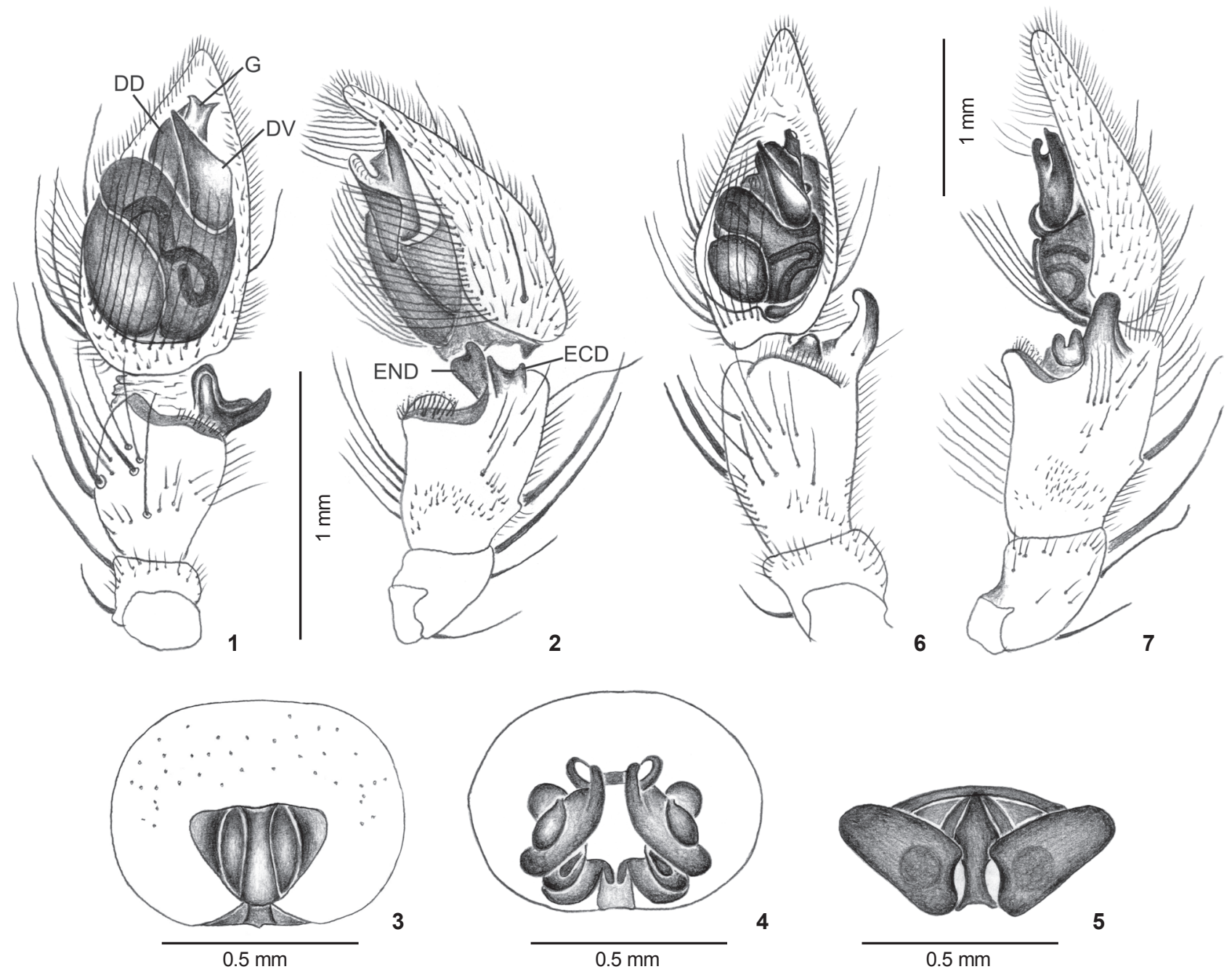

Figures 1-7. (1-5) Syntrechalea neblina sp. nov. (1-2) Male palpus: (1) ventral view; (2) retrolateral view; (3-5) female epigynum: (3) ventral view; (4) dorsal view; (5) posterior view. (6-7) S. robusta sp. nov. (6-7) Male palpus: (6) ventral view; (7) retrolateral view. (DD) Dorsal division of median apophysis, (ECD) ectal division of retrolateral tibial apophysis, (END) ental division of retrolateral tibial apophysis, (G) guide of median apophysis, (VD) ventral division of median apophysis, (HS) head of spermathecae.

tion of the epigynum, but can be distinguished by the presence of two small projections on the middle field of epigynum and the shape of the head of spermathecae (Figs 3-5).

Description. Male (holotype). Total length 6.22. Carapace, 3.32 long, 2.90 wide, yellowish, brownish laterally, slightly flattened. Ocular area dark brown. Clypeus yellowish, 0.26 high. Anterior eye row straight, 0.78 wide; posterior 1.60 wide, recurved. Eye diameters, interdistances, and median ocular quadrangle: AME 0.18, ALE 0.14, PME 0.30, PLE 0.29; AME-AME 0.12, AME-ALE 0.06, PME-PME 0.28, PME-PLE 0.22, OQA 0.44, OQP 0.86, OQH 0.74. Chelicerae yellowish, bristly; promargin and retromargin of fang furrow with three teeth equidistant and equal in size. Sternum yellowish, with scattered setae; 1.07 long, 1.49 wide. Labium yellowish, 0.33 long, 0.49 wide. Legs yellowish with light brown spots on femora, tibia and patella, relative length: IV-II-I, II - femur 7.32/patella-tibia 9.31/metatarsus 7.63/tarsus 5.39/total 29.65; III - 6.25/7.31/8.24/6.65/ 28.45 ; IV - 7.84/9.57/12.36/7.18/36.95. Ventral pairs of macrosetae on tibiae: II-11; III-5; IV-5. Abdomen, 3.73 long, yellowish, with an irregular spot on dorsum and scattered setae; venter yellowish, with scattered setae. Male palpus presenting a prominent median apophysis with a bifurcated guide (Fig. 1). Retrolateral tibial apophysis with the both ental and ectal divisions also bifurcated (Fig. 2). 
Female (paratype). Total length 6.22. Carapace 2.57 long, 2.55 wide, coloration as in male. Clypeus coloration as in male, 0.24 high. Anterior eye row straight, 0.72 wide; posterior 1.52 wide. Eye diameters, interdistances, and median ocular quadrangle: AME 0.13, ALE 0.11, PME 0.28, PLE 0.15; AME-AME 0.16, AME-ALE 0.07, PME-PME 0.34, PME-PLE 0.25, OQA 0.40, OQP 0.88, OQH 0.62. Chelicerae coloration as in male; promargin and retromargin of fang furrow with three teeth equidistant and equal in size. Sternum coloration as in male, 1.55 long, 1.24 wide. Labium coloration as in male, 0.40 long, 0.46 wide. Legs as in male, relative length: IV-I-II-III, I - femur 5.14/patella-tibia 6.47/metatarsus $4.89 /$ tarsus 2.73 /total 19.23; II - 4.81/6.05/4.64/2.90/18.40; III - 3.48/4.31/3.65/2.65/14.09; IV - 5.97/6.48/6.80/3.90/23.15. Ventral pairs of macrosetae on tibiae: I-7; II-7; III-5; IV-4. Abdomen, 3.90 long, coloration as in male. Venter grayish, with numerous white dots and scattered setae. Middle field of epigynum with two small projections, scape slightly projected; spermathecae rounded at apex (Figs 3-5).

Distribution. Known only from the type locality.

Etymology. The specific name is a noun in apposition taken from the Portuguese language reffering to the type locality.

\section{Syntrechalea robusta sp. nov.} Figs 6-11

Types. Male holotype from Palmas, state of Tocantins, Brazil (10¹0’S, 48¹9’W), 11-28.III.1998, M. Calleffo leg., deposited in IBSP 17580. Paratype: one male from Fazenda Globo, Cocalinho, state of Mato Grosso, Brazil (14 $\left.22^{\circ} \mathrm{S}, 50^{\circ} 59^{\prime} \mathrm{W}\right), 01-$ 10.IX.1997, M. Calleffo leg. (IBSP 13895).

Diagnosis. The male of $S$. robusta (Figs 6-10) is similar to the ones of S. syntrechaloides (Mello-Leitão, 1941) by the shape of the median apophysis of male palpus (CARICO 2008: 125, figs 20-21), but can be distinguished by wider and concave ectal division of the retrolateral tibial apophysis (Figs 8-10).

Description. Male (holotype). Total length 7.47. Carapace, 4.15 long, 3.98 wide, light brown, dark brown laterally, slightly flattened. Ocular area dark brown. Clypeus dark brown, 0.44 high. Anterior eye row straight, 1.02 wide; posterior 2.03 wide, recurved. Eye diameters, interdistances, and median ocular quadrangle: AME 0.16, ALE 0.20, PME 0.34, PLE 0.31; AME-AME 0.20, AME-ALE 0.06, PME-PME 0.34, PME-PLE 0.36, OQA 0.52, OQP 1.08, OQH 0.76. Chelicerae dark-brownish, bristly; promargin and retromargin of fang furrow with three teeth equidistant and equal in size. Sternum yellowish, with scattered setae; 1.87 long, 1.76 wide. Labium yellowish, 0.41 long, 0.34 wide. Legs yellowish with light brown spots on femora, tibia and patella, relative length: IV-I-III-II, I - femur 9.31/patella-tibia 12.63/metatarsus 9.44/tarsus 6.65/total 38.03; II - 6.64/7.98/6.63/4.62/25.87; III 7.30/8.64/7.98/3.99/27.91; IV - 9.57/11.72/10.90/7.04/39.23. Ventral pairs of macrosetae on tibiae: I-7; II-8; III-7; IV-5. Abdomen, 3.32 long, dark brown, with numerous dark brown bristles at anterior portion; venter yellowish, with scattered setae. Male palpus with the dorsal division of the median apophysis with a lateral lobe (Figs 6-7). Ectal division of retrolateral tibial apophysis prominent (Figs 7 and 8-10).

Distribution. Brazil (states of Tocantins and Mato Grosso).

Etymology. The specific name is a noun and refers to the prominent ectal division of the retrolateral tibial apophysis of male palpus (Figs 8-10).

\section{New records}

\section{Syntrechalea adis Carico, 2008}

Syntrechalea adis Carico, 2008: 123, figs 2, 4, 14-19 (Male holotype from Igapó, rio Tarumã-Mirim, state of Amazonas, Brazil, 02.III.1983, J. Adis leg., deposited in INPA, not examined).

Distribution. Venezuela, Brazil, Surinam, Peru.

New records. Brazil, Amazonas: São Gabriel da Cachoeira (Pico da Neblina, Bebedouro Velho, $0^{\circ} 46^{\prime} \mathrm{N}, 65^{\circ} 59^{\prime} \mathrm{W}$ ), 1 female, 13.X.2007, D. Candiani leg. (INPA); Novo Airão, Estação Ecológica de Anavilhanas ( $\left.2^{\circ} 37^{\prime} \mathrm{S}, 60^{\circ} 56^{\prime} \mathrm{W}\right), 2$ females, VIII.2006, A.J. Santos leg. (IBSP 73509); Pará: Oriximiná, Igarapé

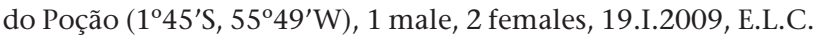
Silva leg. (MCTP).

\section{Syntrechalea brasilia Carico, 2008}

Syntrechalea brasilia Carico, 2008: 128, figs 2, 7, 28-31 (Male holotype from Distrito Federal, Brasília, Brazil, XI.2003, M. Prada leg., deposited in the Departamento de Zoologia, in the Universidade de Brasília, not examined).

Distribution. Brazil (states of Rondônia, Distrito Federal, and Minas Gerais).

New records. Brazil, Rondônia: Vilhena $\left(12^{\circ} 43^{\prime} \mathrm{S}, 60^{\circ} 08^{\prime} \mathrm{W}\right)$, 1 female, IX.1999, M. Carvalho leg. (IBSP 81005); Minas Gerais: Uberlândia (18 $54^{\prime}$ S, $\left.48^{\circ} 15^{\prime} \mathrm{W}\right), 1$ male, XI.1996, D. Cunha leg. (IBSP 8334); (Reserva de Caça e Pesca, 18 $54^{\circ}$ S, 48 ${ }^{\circ} 18^{\prime} \mathrm{W}$ ), 1 male, XI.1996, D. Cunha leg. (IBSP 8333).

\section{Syntrechalea caporiacco Carico, 2008}

Syntrechalea caporiacco Carico, 2008: 127, figs 2, 24-27 (Male holotype from Base Cerro Yapacama, Amazonas, Venezuela, 23.II.1978, C.W. Myers leg., deposited in American Museum of Natural History, not examined).

Distribution. Venezuela, Brazil, Guyana, Peru.

New records. Brazil, Bahia: Una (Reserva Biológica de Una, $\left.15^{\circ} 16^{\prime} \mathrm{S}, 39^{\circ} 04^{\prime} \mathrm{W}\right), 1$ male, 1 female, 15-28.XI.2000, A.D. Brescovit et al. leg. (IBSP 45516, 47674); Alagoas: Maceió (Serra da Saudinha, Ipioca, 9³9'S, 35²4 'W), 1 female, XI.2004, G.Q.C. Correia leg. (IBSP 3835).

\section{Syntrechalea napoensis Carico, 2008}

Syntrechalea napoensis Carico, 2008: 129, figs 32, 33 (Male holotype from Napo, Alianhui, $20 \mathrm{~km}$ E of Puerto Napo, Ecuador, $01^{\circ} 00^{\prime} \mathrm{S}, 77^{\circ} 25^{\prime} \mathrm{W}$, IX.1996, E.S. Ross leg., deposited in the California Academy of Sciences, not examined). 

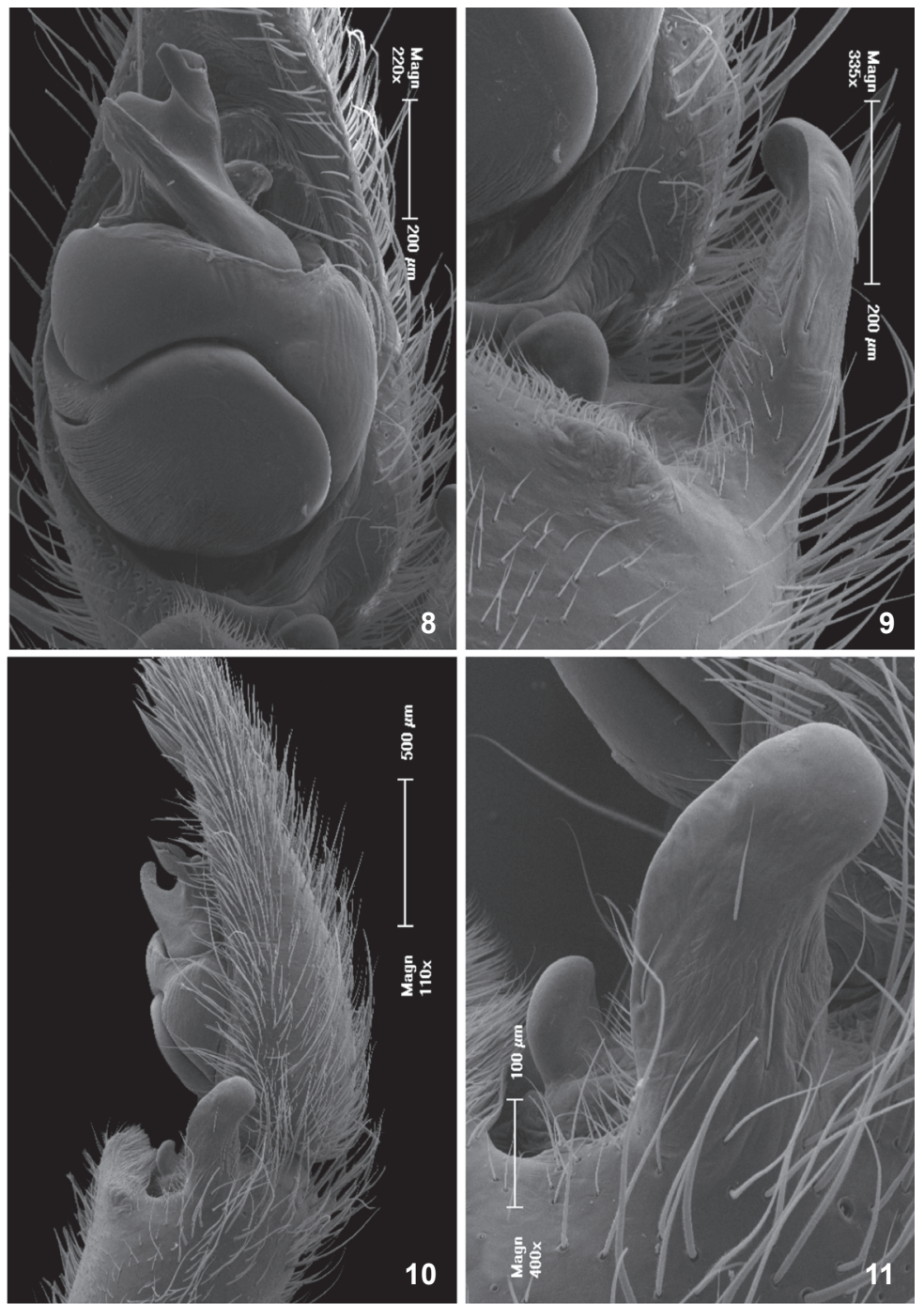

Figures 8-11. Syntrechalea robusta sp. nov. , male palpus: (8) detail of bulbus, ventral view; (9) detail of retrolateral tibial apophysis, ventral view; (10) male palpus, lateral view; (11) detail of ectal division of retrolateral tibial apophysis, lateral view.

Distribution. Ecuador, Brazil.

New record. BraziL, Acre: Senador Guiomard (Reserva Extrativista de Catuaba, $\left.10^{\circ} 08^{\prime} \mathrm{S}, 67^{\circ} 43^{\prime} \mathrm{W}\right), 1$ male, 2002, E.F. Morato leg. (IBSP 84628).

\section{Syntrechalea syntrechaloides (Mello-Leitão, 1941)}

Trechalea syntrechaloides Mello-Leitão, 1941: 246 (Female holotype from Cachoeirinha, Bocaiúva, state of Paraná, Brazil, no date, L. De Morrietes leg., deposited in Museu Nacional do Rio de Janeiro, not examined).

Syntrechalea syntrechaloides; Carico, 2008: 127, figs 2, 5, 20-23.

Distribution. Colombia, Venezuela, Brazil, Guyana, Peru, Bolivia.

New records. BRAzIL, Acre: Xapurí (Reserva Extrativista da
Pimenteira, $10^{\circ} 36^{\prime}$ S, 68 $29^{\prime}$ W), 1 female, 05-07.IV.1996, Equipe IBSP/SMNK leg. (IBSP 16029); Amazonas: Manaus (Reserva km $\left.41,3^{\circ} 06^{\prime} \mathrm{S}, 60^{\circ} 01^{\prime} \mathrm{W}\right), 1$ male, VIII.2000, A.J. Santos leg. (IBSP $39938)$; Presidente Figueiredo $\left(2^{\circ} 01^{\prime} \mathrm{S}, 60^{\circ} 01^{\prime} \mathrm{W}\right)$, Usina Hidrelétrica de Balbina ( $\left.1^{\circ} 30^{\prime} \mathrm{S}, 59^{\circ} 12^{\prime} \mathrm{W}\right), 1$ female, 1987/1988, Equipe IBSP leg. (IBSP 10861); Pará: Santarém (Campus do Aeroporto, $\left.2^{\circ} 25^{\prime} \mathrm{S}, 54^{\circ} 42^{\prime} \mathrm{W}\right), 1$ male, 28.I.1994, A.D. Brescovit leg. (IBSP 62931); Oriximiná (Igarapé do Poção, $1^{\circ} 45^{\prime}$ S, $\left.55^{\circ} 49^{\prime} \mathrm{W}\right), 1$ male, 1 female, 19.I.2009, E.L.C. Silva leg. (MCTP); Vitória do Xingu ( $\left.2^{\circ} 52^{\prime} \mathrm{S}, 52^{\circ} 01^{\prime} \mathrm{W}\right), 1$ male, 28.XI.2008 (MPEG 4781); Paraíba: São José da Mata (Sítio São Miguel, $7^{\circ} 12^{\prime}$, $35^{\circ} 55^{\prime} \mathrm{W}$ ), 2 males, 10.IV.1997, A.D. Brescovit leg. (IBSP 8893); Goiás: Catalão (18¹0’S, 4756’W), 4 males, 19-28.IX.1999, G.G. Montingelli leg. (IBSP 26242); São Paulo: Rio Claro (22²4'S, 
$47^{\circ} 34^{\prime}$ W), 2 males, 1 female, 04.XI.2008, A.M. Giroti \& E. Paula leg. (IBSP 122267, 122737, 122485); 2 females, 17.XII.2008 (IBSP 122483, 122484); Paraná: Foz do Iguaçu (Parque Nacional de Foz do Iguaçu, $\left.25^{\circ} 36^{\prime} \mathrm{S}, 54^{\circ} 25^{\prime} \mathrm{W}\right), 1$ female, 03-12.III.2002, Equipe Biota leg. (IBSP 52625); Rio de Janeiro: Pinheiral (Fazenda Santa Helena, $22^{\circ} 34^{\prime}$ S, $44^{\circ} 21^{\prime} \mathrm{W}$ ), 1 female, 05-11.XI.1999, A.D. Brescovit leg. (IBSP 52733).

\section{Syntrechalea tenuis F.O.Pickard-Cambridge, 1902}

Syntrechalea tenuis F.O.Pickard-Cambridge, 1902: 314 (Female holotype from Bugaba, Chiriquí, Panama, Champion leg., deposited in The Natural History Museum, London, not examined); Roewer, 1954: 139; Bonnet, 1956: 4225; Carico, 2008: 120 , figs $1,8-11$.

Syntrechalea porshi Reimoser, 1939: 339 (Male holotype from Rio Reventatou, Hamburg Farm, Limon, 1930, E. Reimoser leg., deposited in Naturhistorisches Museum zu Hamburg, not examined); Roewer, 1954: 139; Platnick, 2009; Carico, 2008: 120 (Syn.).

Distribution. Mexico to Colombia.

New records. BRAzIL, Acre: Xapurí (Reserva Extrativista da Pimenteira, $\left.10^{\circ} 36^{\prime} \mathrm{S}, 68^{\circ} 29^{\prime} \mathrm{W}\right), 1$ male, 1 female, 05-07.IV.1996, Equipe IBSP/SMNK leg. (IBSP 16086); Amazonas: Manaus (Reserva km 41, 306'S, 6001'W), 2 males, VII-VIII.2006, A.J. Santos leg. (IBSP 80256); Bahia: Salvador (Parque Municipal do Pituaçu, $12^{\circ} 59^{\prime} \mathrm{S}, 38^{\circ} 29^{\prime} \mathrm{W}$ ), 2 males, 20-25.I.2004, E.S.S. Álvares leg. (IBSP 80787); Alagoas: Maceió (Serra da Saudinha, Ipioca, $9^{\circ} 39^{\prime} \mathrm{S}, 35^{\circ} 44^{\prime} \mathrm{W}$ ), 1 male, 04.II.2004, G.Q.C. Correia leg. (IBSP 63811); Pará: Vitória do Xingu ( $\left.2^{\circ} 52^{\prime} \mathrm{S}, 52^{\circ} 01^{\prime} \mathrm{W}\right), 1$ male, 25.IX.2000 (MPEG 4787); São Paulo: Rio Claro $\left(22^{\circ} 24^{\prime}\right.$ 'S, $47^{\circ} 34^{\prime}$ W), 1 female, 20.I.2006, A.M. Giroti \& E. Paula leg. (IBSP 59115).

\section{ACKNOWLEDGMENTS}

We wish to thank Antonio D. Brescovit (IBSP), Célio Magalhães (INPA) and Alexandre B. Bonaldo (MPEG) for the loan of the material. The staff of Centro de Microscopia e
Microanálises (CEMM/PUCRS) for the SEM images. This study was supported by Conselho Nacional de Desenvolvimento Científico e Tecnológico (process 140282/2008-4 for ELCS).

\section{LITERATURE CITED}

BonNet, P. 1956. Bibliographia araneorum. Analyze méthodique de toute la littérature aranéologique jusqu'en 1939. Toulouse, Les Fréres Douladore, vol. 2, part 2, p. 919-1926.

CARICO, J.E. 1993. Revision of the genus Trechalea Thorell (Araneae, Trechaleidae) with a review of the taxonomy of the Trechaleidae and Pisauridae of the Western Hemisphere. Journal of Arachnology 21 (3): 226-257.

CARICO, J.E. 2008. Revision of the Neotropical arboreal spider genus Syntrechalea (Araneae, Lycosoidea, Trechaleidae). Journal of Arachnology 36 (1): 118-130.

Mello-Leitão, C. 1941 Aranhas do Paraná. Archivos do Instituto de Biologia de São Paulo 11: 235-257.

Platnick, N.I. 2009. The World Spider Catalog version 10.0. New York, American Museum of Natural History, available on line at: http://research.amnh.org/entomology/spiders/ catalog/html [Access: 03.VIII.2009].

Pickard-Cambridge, F.O. 1902. Arachnida - Araneida and Opiliones. Biologia Centrali-Americana, Zoology, 2: 313-424.

Reimoser, E. 1939. Wissenschaftliche Ergebnisse der osterreichischen biologischen Expedition nach Costa Rica. Die Spinnenfauna. Annalen des Natuhistoreschen Museums in Wien 50: 328-386.

Roewer, C.F. 1954. Katalog der Araneae. Bruxelles, Institut Royal des Sciences Naturelles de Belgique, vol. 2a, 923p.

SiLvA, E.L.C. \& A.A. Lise 2008. Description of a new species of the spider genus Syntrechalea (Araneae, Lycosoidea, Trechaleidae) from Colombia. Revista Brasileira de Zoologia 25 (3): 495-498.

Silva, E.L.C. \& A.A. Lise. 2009. On the taxonomy of Trechaleidae (Araneae: Lycosoidea) from Colombia and Peru. Zoologia 26 (2): 357-362.

Submitted: 03.VIII.2009; Accepted: 03.III.2010.

Editorial responsibility: Antonio Domingos Brescovit 